Daran hat sich bis heute nichts geändert, wie die Inhaber stolz berichten. Ganz wesentlich trägt dazu ihr persönliches Umfeld aus Bekannten und Freunden bei, die den ambulanten Dienst von Anfang an in ihrem Netzwerk weiter empfahlen.

\section{Eigenverantwortlich, anders, besonders}

Die Gründer haben sich auf die Fahnen geschrieben, in ihrem ambulanten Dienst die Pflege nach ihren Vorstellungen zu gestalten. Was heißt das konkret für die Klienten und die Menschen, die bei AmbuPlus arbeiten? Zunächst einmal sind die Inhaber jederzeit ansprechbar und mitten im Geschehen, „Chefs zum Anfassen“ - darauf legen beide Wert. Der Dienst soll „klein, aber fein“ bleiben, denn Klienten und Mitarbeiter schätzen die persönliche und familiäre Atmosphäre bei AmbuPlus. Es gibt keine starren Einsatzzeiten. Die Arbeitszeiten werden bewusst familienfreundlich geplant. Pflegende und Klienten profitieren von der größeren Flexibilität. Die Touren sind von vornherein großzügig geplant, so dass die Pflegenden ausreichend Zeit haben für ihre Klienten und ohne Zeitdruck arbeiten können. Bestimmte Leistungen wie Erstgespräche oder die 24h-Rufbereitschaft werden ausschließlich durch die Inhaber erbracht. Diese sind auch Seelentröster und Betreuer „für alles“ wie Silvia Gerhard sagt und geben Hilfestellung beispielsweise beim Schriftverkehr mit den Kassen bei Patienten, die keine Angehörige haben. In vertretbarem Rahmen fallen dann auch mal Leistungen an, die sie nicht abrechnen können. Das sei eine zeitliche Mischkalkulation: „Bei einem Klienten braucht man mehr Zeit, beim anderen weniger. Am Ende passt es wieder."

Das Team wird sehr sorgfältig danach ausgewählt, ob die Personen zueinander passen. Bis auf die Hauswirtschafterinnen sind beispielsweise alle zwischen Ende 20 und Mitte 30. Die „Chemie“ im Team stimme. Was nach Meinung der AmbuPlus-Inhaber ein gutes Team ausmacht lesen Sie im Kasten. Entscheidend ist - neben der fachlichen Qualifikation - der erste Eindruck: Sympathie, Offenheit, Motivation und ganz wichtig: der Wille zur stetigen Weiterbildung und Qualifizierung.

Seine MitarbeiterInnen findet Ambu Plus über persönliche Kontakte, Mundpropaganda und herkömmliche Stellenausschreibungen. Ab Herbst 2016 will der Pflegedienst selbst ausbilden.

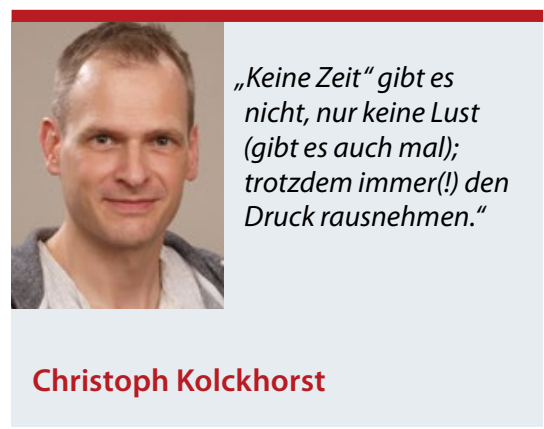

\section{Gute Aussichten}

Vieeeel Idealismus, viel Mut, Verrücktheit, Selbstsicherheit und Selbstbewusstsein brauche man als Gründer im Pflegebereich. Dennoch: Die Zukunftsperspektiven von Pflegediensten schätzen Gerhard und Kolckhorst generell als positiv ein. Der demografische Wandel sorge für eine dauerhaft gute Auftragslage, da die meisten Menschen die Pflege zu Hause einem Pflegeheim vorzögen. Und wie sieht es mit der Versorgungsqualität in Zeiten knapper Ressourcen aus? Darauf hat Silvia Gerhard eine klare Antwort: „Mit dem richtigen Team und guter Organisation ist das wirtschaftliche Betreiben eines Pflegedienstes unter den vorhandenen Rahmenbedingungen möglich." Sabine M. Kempa

\title{
DAS MACHT EIN GUTES TEAM AUS
}

\section{AUF EINEN BLICK}

\section{MOTIVATION}

Ein Team braucht eine motivierende Aufgabe. Alle müssen sie als sinnvoll empfinden. Motivation ist die Dankbarkeit unserer Patienten und die Wertschätzung der Mitarbeiter durch den Arbeitgeber

\section{IDENTIFIKATION}

Jedes Mitglied muss sich mit dem Team identifizieren, alle an einem Strang ziehen. Basis sind gemeinsame Erfahrungen und gemeinsame Werte.

\section{TEAMZUSAMMENSTELLUNG}

Die Zusammenstellung eines Teams ist entscheidend für den Erfolg. Dabei dürfen nicht nur fachliche Kriterien gelten. Die Teamplayer müssen auch die Fähigkeit und den Willen haben, sich gegenseitig zu unterstützen.

\section{LEISTUNGSNIVEAU}

Alle im Team müssen sich auf einem ähnlichen Leistungsniveau begegnen. So entsteht ein Wettbewerb, der alle antreibt, noch besser zu werden.

\section{AUFGABENVERTEILUNG}

Muss klar geregelt sein, Überforderungen müssen vermieden, Belastungen gerecht verteilt werden.

\section{TEAMKLIMA}

Offene Kommunikation, in der neue Ideen belohnt und Fehler toleriert werden.

\section{TEAMFÜHRUNG}

Alle Stimmen ernst nehmen, die Verantwortung aber behalten und Entscheidungen selbst fällen.

(Silvia Gerhard, Christoph Kolckhorst)

\section{AMBU PLUS GbR \\ Inhaber: Silvia Gerhard, Christoph Kolckhorst}

Gründung: 2013 mit drei Vollzeitbeschäftigten ( 2 Inhaber + 1 Angestellte)

Aktuell: Neben den Inhabern 5 examinierte Krankenschwestern/-pfleger, (4 festangestellt, 1 GfB), 7 Betreuungskräfte, 2 Hauswirtschafterinnen

Schwerpunkt: Palliativpflege, Intensivpflege, Pflege von Patienten mit chronischen Wunden. Grundsätzlich alle von den Krankenkassen zugelassenen Leistungen gemäß SGB V und VI

www.ambu-plus.de 